\title{
TESINA
}

\section{Conocimiento, prácticas y aptitudes del profesional de enfermería en el manejo de pacientes con enfermedad pulmonar obstructiva crónica internados en el Instituto Nacional de Enfermedades Respiratorias y del Ambiente}

\author{
Edmundo Octavio Pedrozo Cáceres, María Francisca Godoy Báez
}

\begin{abstract}
Resumen
Introducción: EPOC es una enfermedad incurable y puede producir daños severos e irreversibles. Caracterizada por una alta complejidad que requiere una importante especialización en los cuidados. El conocimiento sobre el cuidado permite una mejor atención al paciente, a favor de su más rápida recuperación o disminución de los síntomas para una mejor calidad de vida de los pacientes.
\end{abstract}

Objetivo General: Identificar los conocimientos, las prácticas y las aptitudes del profesional de enfermería en el manejo del paciente con EPOC, internado en el Instituto Nacional de Enfermedades Respiratorias y del Ambiente.

Material y Método: Estudio no experimental, pues no hay intervención en las variables, tiene un enfoque cuantitativo, pues los datos son expresados en forma numérica. Se elaboró una encuesta para recolectar los datos, se tuvo en cuenta bibliografía que refiera sobre el manejo del paciente con EPOC.

Resultados: Nivel de conocimientos, 33,3\% del profesional de enfermería tiene un conocimiento "aceptable" y el " $16,7 \%$ " tiene un nivel insuficiente. Teniendo en cuenta los resultados, se considera que el $80 \%$ tiene conocimiento "suficiente" sobre la EPOC. Nivel de práctica, el 62,5\% de los profesionales tienen prácticas insuficientes o no adecuadas y el 16,7\% excelente. Considerando como 70\% de prácticas respondidas adecuadamente el 78,6\% del profesional no tiene prácticas adecuadas en el manejo de estos pacientes. En cuanto a las aptitudes, se observó aptitudes neutras,

Conclusiones: 3 de cada 10 personales de enfermería tienen conocimientos suficientes. 2 de cada 10 personales de enfermería tienen prácticas adecuadas. Se observó aptitudes neutras entre los participantes.

Palabras clave: conocimientos, prácticas, aptitudes.

1. UNIBE. Facultad de Ciencias de la Salud. Carrera de Enfermería.

Tesina para obtener el grado de Lic. en Enfermería.

E-mail: pedrozooctavio@gmail.com

DOI: $10.26885 /$ rcei.foro.2018.180 
Manejo de pacientes con enfermedad pulmonar obstructiva crónica.Pedrozo \& Godoy

\section{RefERENCIAS}

González Juárez, F (2016). Diagnóstico y Tratamiento en Neumología. Editorial El Manual Moderno.

Gumucio, S. (2011). Recogida de datos cuantitativos Ejemplos de encuestas CAP. Francia.

Moorhead, S. (2009). Clasificación de Resultados de Enfermería (NOC). Madrid, España: Elsevier.

NANDA International. (2011). Diagnósticos enfermeros definiciones y clasificación. Madrid: Elsevier.

Pereles, M. A., González, C. (2008). Plan de cuidados estandarizados Procesos EPOC. 